\title{
ЕКСПЕРИМЕНТАЛЬНО-ТЕОРЕТИЧНІ ПІДХОДИ ДО СТВОРЕННЯ КОМП'ЮТЕРНОЇ ПРОГРАМИ ДЛЯ ВІРТУАЛЬНОГО СКРИНІНГУ СКАВЕНДЖЕРІВ NО В РЯДУ АЗАГЕТЕРОЦИКЛІВ
}

\author{
В. П. Риженко, І. Ф. Бєленічев, \\ О. А. Рижов, С. В. Левіч
}

Запорізький державний медичний університет

\begin{abstract}
Створення комп'ютерних програм для віртуального скринінгу є актуальним завданням для сучасної фрармакології і медицини. У статті показані деякі експериментально-теоретичні підходи до створення комп'ютерної програми для віртуального скринінгу скавенджерів NO в ряду азагетероциклів. Наведено методи оцінки антиоксидантної активності, квантово-механічні та статистичні розрахунки. Розглянуто перспективи і переваги комп'ютерного моделювання активності сполук.
\end{abstract}

Ключові слова: віртуальний скринінг, дескриптори, антиоксидантна активність.

\section{EXPERIMENTAL AND THEORETICAL APPROACHES TO THE CREATION OF COMPUTER PROGRAM FOR VIRTUAL SCREENING OF SCAVENGERS NO IN A RANGE OF AZAGETEROCYCLES}

\author{
V. P. Ryzhenko, I. F. Belenichev, \\ O. A. Ryzhov, S. V. Levich
}

Zaporozhye State Medical University

\begin{abstract}
The creation of computer programs for virtual screening is an urgent task for modern pharmacology and medicine. The article shows some experimental and theoretical approaches to the creation of a computer program for virtual screening of scavengers NO in a range of azageterocycles. Methods for estimating antioxidant activity, quantum mechanical and statistical calculations are presented. Prospects and advantages of computer simulation of the activity of compounds are considered.
\end{abstract}

Key words: virtual screening, descriptors, antioxidant activity.

\section{ЭКСПЕРИМЕНТАЛЬНО-ТЕОРЕТИЧЕСКИЕ ПОДХОДЫ К СОЗДАНИЮ КОМПЬЮТЕРНОЙ ПРОГРАММЫ ДЛЯ ВИРТУАЛЬНОГО СКРИНИНГА СКАВЕНДЖЕРОВ NО В РЯДУ АЗАГЕТЕРОЦИКЛОВ}

\begin{abstract}
В. П. Рыженко, И. Ф. Беленичев, А. А. Рыжов, С. В. Левич

Запорожский государственный медицинский университет

Создание компьютерных программ для виртуального скрининга является актуальной задачей для современной фрармакологии и медицины. В статье показаны некоторые экспериментально-теоретические подходы к созданию компьютерной программы для виртуального скрининга скавенджеров NO в ряду азагетероциклов. Приведены методы оценки антиоксидантной активности, квантово-механические и статистические расчеты. Рассмотрены перспективы и преимущества компьютерного моделирования активности соединений.
\end{abstract}

Ключевые слова: виртуальный скрининг, дескрипторы, антиоксидантная активность. 
Введение. В последнее время мы являемся свидетелями бурного развития технологий «виртуального» скрининга — компьютерного моделирования активности соединений. Подобные методы позволяют прогнозировать биологическую активность как уже имеющихся коллекций соединений, так и еще не существующих в природе веществ, то есть еще до синтеза прогнозировать их возможности по воздействию на живые организмы. Учитывая тот факт, что современная фармакология оперирует большим количеством видов биологической активности, предсказание профиля активности соединений имеет особое значение. Был осознан тот факт, что неограниченный рост размеров синтезированных и тестируемых библиотек соединений не приводит сам по себе к интенсификации исследовательских программ, но даже способен приводить к неоправданным дополнительным затратам [6]. Возникла и постепенно приобретает все большую практическую значимость относительно молодая дисциплина — компьютерный, или виртуальный скрининг (от англ. virtual screening виртуальный отбор, проверка) [7].

Цель исследования: описание экспериментальнотеоретических подходов к созданию компьютерной программы для виртуального скрининга NO в ряду азагетероциклов.

Материал и методы исследования. Проведены квантово-механические расчеты дескрипторов [3] HOMOEnergy (высшая занятая молекулярная орбиталь) и LUMOEnergy (низшая вакантная молекулярная орбиталь) при помощи программного комплекса WinMopac (v. 7.2). Оптимизация структуры достигалась использованием полуэмпирического метода AM1 с такими параметрами: Calculation = SinglePoint, WaveFunction $=$ ClosedShell $(\mathrm{RHF})$. Показатель реактивного индекса рассчитывали по формуле:

$$
\omega=\chi^{2} / 2 \eta \text {. }
$$

Также проведена оценка антиоксидантной активности (AОА) [2]. Метод основан на фотоиндукции нитропруссида натрия, сопровождающейся накоплением NO-радикала [5]. Далее показатели AOA рассчитывали по формуле:

$$
A O A=\frac{E_{t}-E_{C}}{E_{C}} \times 100 \%
$$

где $E_{t}$ - оптическая плотность испытуемого образца, $E_{c}$ - оптическая плотность контрольного образца.
Статистический анализ. Анализ статистических данных проводили с помощью программного обеспечения STATISTICA 6.0 для Windows [1]. Данные представлены в виде среднего значения для образца \pm стандартная ошибка среднего. Верность различий между экспериментальными группами оценивали с помощью критерия Стьюдента и критерия Фишера.

Результаты и их обсуждение. Квантовомеханические расчеты показали, что реактивный индекс исследуемых соединений находится в пределе от -1,9605 эВ до -2,9398 эВ [4]. Показатели HOMO и LUMO данных соединений также находятся на высоком уровне. Исследуемые соединения [4] показали высокие показатели АOA и в большинстве случаев превышали стандарт. Показатели АОА исследуемых соединений находятся в пределах 49,43-82,16\% (концентрация 10-3 моль/л). При концентрации 10-5 моль/л показатели АОА почти всех соединений уменьшились, но превышали стандарт. При снижении концентрации до 10-7 моль/л происходило уменьшение антиоксидантных свойств, но все соединения показывали активность, которая превосходила стандартный показатель.

Таким образом, при исследовании соединений было выявлено, что все они обладают антиоксидантными свойствами in vitro на модели фотоиндукции нитропруссида натрия благодаря свойствам скавенджеров NO. При сравнении значений рассчитанных дескрипторов с полученными данными in vitro удалось установить линейную зависимость AOA от значений HOMO и LUMO. В ходе анализа результатов исследования нами было установлено, что АОА изучаемых соединений находится в прямой зависимости от реактивного индекса (рис. 1). В связи с этим было решено проводить определение вероятной активности соединений, основываясь на линейной интерполяции.

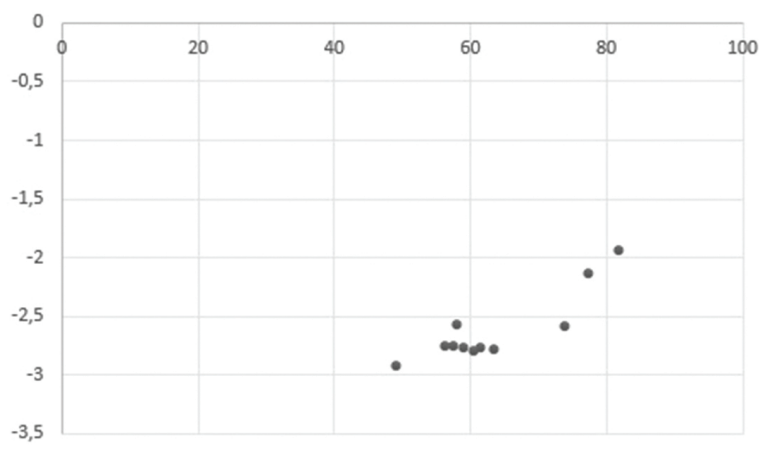

Рис. 1. Зависимость АОА от реактивного индекса 


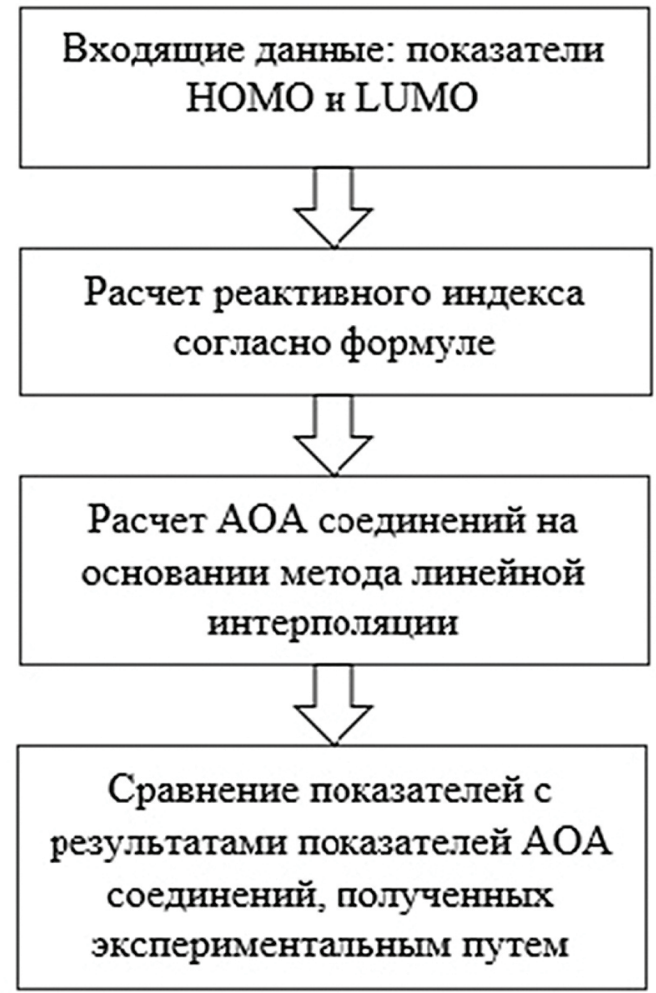

Рис. 2. Алгоритм для компьютерной программы виртуального скрининга

Полученные результаты могут быть использованы для дальнейшего поиска NO-скавенджеров в ряду азагетероциклов и использования их индекса реактивности в качестве маркера антиоксидантных свойств. В ходе анализа всех полученных данных был предложен алгоритм для компьютерной программы виртуального скрининга (рис. 2).

Программу лучше всего реализовать в виде вебприложения - это решение поможет сократить время тестирования программы. В дальнейшем эта реализация привлечет большее количество пользователей, чем стационарная версия.

\section{Литература.}

1. Зайцев В. М. Прикладная медицинская статистика : учебное пособие / В. М. Зайцев, В. Г. Лифляндский, В. И. Маринкин. - СПб : Фолиант, 2006. — 432 с.

2. An effective antioxidant drug on prevention of the necrosis of zone of stasis: N-acetylcysteine / M. Deniz, H. Borman, T. Seyhan, M. Haberal // Burns. - 2013. — Vol. 39, No. 2. - P. 320-325.

3. Estimation of relationship between the structure of trihaloacetylazulene derivatives determined by a semiempirical molecular-orbital method (PM5) and their cytotoxicity / M. Ishihara, H. Wakabayashi, N. Motohashi, H. Sakagami // Anticancer Res. - 2010. — Vol. 30, No. 3. — P. 837-842.

\section{Выводы.}

Полученые эксперементальным путем данные об АОА соединений и данные расчетов дескрипторов (HOMO и LUMO) позволяют установить прямую зависимость АОА от реактивного индекса. В результате этого возможно проводить предсказание АОА на основании метода линейной интерполяции. Создание компьютерной программы для виртуального скрининга существенно сократит время и средства при исследовании новых синтезируемых соединений.

4. Study of dependence of xanthine derivatives NOscavenger properties from energy descriptors / V. P. Ryzhenko, O. A. Ryzhov, I. F. Belenichev, S. V. Levich // Biological Markers and Guided Therapy. — 2018. - Vol. 5, No. 1. — P. 37-46.

5. Vanin A. F. Dinitrosyl iron complexes and S-nitrothiols are two possible forms of stabilization and transport of nitric oxide in biological systems / A. F. Vanin // Biochemistry (Mosc). - 1998. - Vol. 63, No. 7. P. 782-793.

6. Virtual screening for bioactive molecules / H. J. Bohm, G. Schneider (Eds.). — Weinheim : Wiley-VCH, 2000. -308 p. 
7. Walters W. P. Virtual screening - an overview / W. P. Walters, M. T. Stahl, M. A. Murcko // Drug Disc. Today. - 1998. — Vol. 3, No. 4. - P. 160-178.

\section{References.}

1. Zaitsev, V. M., Liflyandskii, V. G., \& Marinkin, V. I. (2006). Prikladnaya meditsinskaya statistika [Applied Medical Statistics]: textbook. St. Petersburg: Foliant. [In Russian].

2. Deniz, M., Borman, H., Seyhan, T., \& Haberal, M. (2013). An effective antioxidant drug on prevention of the necrosis of zone of stasis: N-acetylcysteine. Burns, 39(2), 320-325. doi: 10.1016/j.burns.2012.06.015.

3. Ishihara, M., Wakabayashi, H., Motohashi, N., Sakagami, H. (2010). Estimation of relationship between the structure of trihaloacetylazulene derivatives determined by a semiempirical molecular-orbital method (PM5) and their cytotoxicity. Anticancer Res., 30(3), 837-842.
4. Ryzhenko, V. P., Ryzhov, O. A., Belenichev, I. F., \& Levich, S. V. (2018). Study of dependence of xanthine derivatives NO-scavenger properties from energy descriptors. Biological Markers and Guided Therapy, 5(1), 37-46. doi: 10.12988/bmgt.2018.857.

5. Vanin, A. F. (1998). Dinitrosyl iron complexes and S-nitrothiols are two possible forms of stabilization and transport of nitric oxide in biological systems, Biochemistry (Mosc)., 63(7), 782-793.

6. Bohm, H. J., \& Schneider, G. (Eds.) (2000). Virtual screening for bioactive molecules. Weinheim: WileyVCH. doi: 10.1021/ja0152052.

7. Walters, W. P., Stahl, M. T., \& Murcko, M. A. (1998). Virtual screening - an overview. Drug Disc. Today, 3(4), 160-178. doi: 10.1016/S1359-6446(97)01163-X. 